\title{
UNIVERSAL QUADRATIC FORMS AND WHITNEY TOWER INTERSECTION INVARIANTS
}

\author{
JAMES CONANT, ROB SCHNEIDERMAN, AND PETER TEICHNER \\ Dedicated to Mike Freedman on the occasion of his 60th birthday.
}

\begin{abstract}
We first remind the reader of a simple geometric description of the KirbySiebenmann invariant of a 4-manifold in terms of a quadratic refinement of its intersection form. This is the first in a sequence of higher-order intersection invariants of Whitney towers, studied by the authors, particularly for the 4-ball.

In the second part of this paper, a general theory of quadratic forms is developed and then specialized from the non-commutative to the commutative to finally, the symmetric settings. The intersection invariant for twisted Whitney towers is shown to be the universal symmetric refinement of the framed intersection invariant. As a corollary we obtain a short exact sequence that has been essential in the understanding of Whitney towers in the 4-ball.
\end{abstract}

\section{INTRODUCTION}

A beautiful consequence of Mike Freedman's disk embedding theorem is the existence of non-smoothable 4-manifolds. In the easiest setting, his result can be stated as follows.

Theorem 1. Any odd unimodular symmetric form $\lambda: \mathbb{Z}^{m} \otimes \mathbb{Z}^{m} \rightarrow \mathbb{Z}$ is realized as the intersection form of exactly two closed simply-connected oriented 4-manifolds (up to homeomorphism). These 4-manifolds are homotopy equivalent and are distinguished by the following (equivalent) criteria: Exactly one of the manifolds...

(i) ... is smoothable after crossing with $\mathbb{R}$.

(ii) ... is smoothable after connected sum with finitely many copies of $S^{2} \times S^{2}$.

(iii) ... has a linear reduction of its micro normal bundle.

(iv) ... has vanishing Kirby-Siebenmann invariant in $\mathbb{Z}_{2}$.

(v) ... exhibits the following formula for a quadratic refinement $\tau$ of $\lambda$ :

$$
\tau(c) \equiv \frac{\lambda(c, c)-\text { signature }(\lambda)}{8} \quad \bmod 2 \quad \forall \text { characteristic elements } c .
$$

Remark 2. By Donaldson's Theorem A [5], exactly the diagonalizable odd forms $\lambda$ are realized by closed smooth 4 -manifolds. Diagonal forms are realized by connected sums of complex projective planes (with varying orientations); in fact, most such forms are now known to admit infinitely many smooth representatives (all being homeomorphic by the above theorem), see e.g. [8].

Criterion (v) is the most elementary and will be explained in detail in Section 2. The key is the following geometric interpretation for the quadratic refinement $\tau(c)$. In a simplyconnected closed 4-manifold $M$, any class in $H_{2}(M)$ can be represented by a (topologically

Key words and phrases. Whitney towers, twisted Whitney towers, quadratic refinements, Arf invariants, Lie algebra. 
generic) immersed sphere $S: S^{2} \rightarrow M$. This means that $S$ looks locally like $\mathbb{R}^{2} \times 0 \subset \mathbb{R}^{4}$, except for finitely many double points around which $S$ looks like $\mathbb{R}^{2} \times 0 \cup 0 \times \mathbb{R}^{2} \subset \mathbb{R}^{4}$. One can add more local self-intersection points to $S$ until their algebraic sum is zero. This implies that one can choose Whitney disks $W_{i}$, pairing all these self-intersection points. These are (topologically generic) immersed disks $W_{i}: D^{2} \rightarrow M$ whose boundary consists of two arcs, each going between the two intersection points but on different sheets, see Figure 1 .
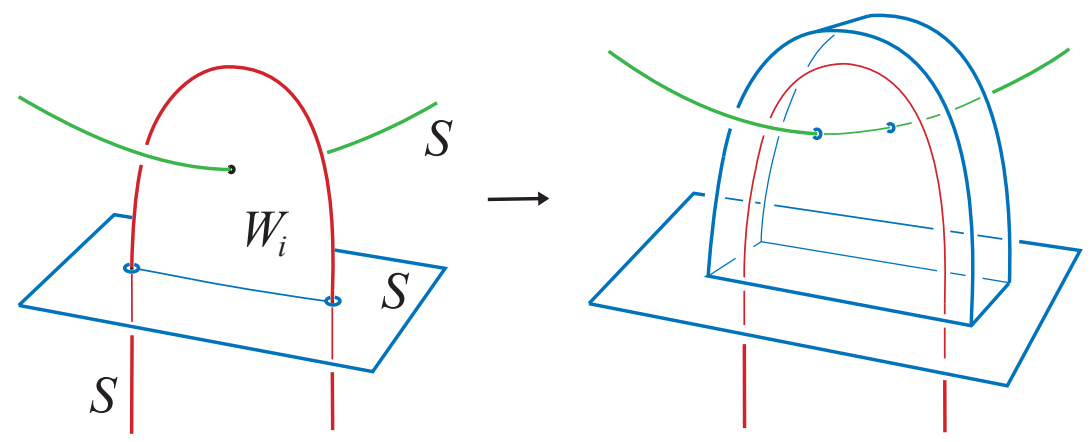

Figure 1. A (framed) Whitney disk and a Whitney move.

We will explain in Lemma 4 why $\tau(c)$ equals an intersection invariant $\tau_{1}\left(S, W_{i}\right)$, computed by summing the (topologically generic) intersections between an immersed sphere $S$, representing the characteristic $c \in H_{2}(M)=\mathbb{Z}^{m}$, and (the interiors of) framed Whitney disks $W_{i}$ for $S$ :

$$
\tau(c)=\tau_{1}\left(S, W_{i}\right):=\sum_{i} \#\left\{S \pitchfork W_{i}\right\} \quad \bmod 2
$$

In [7, this invariant was called the Kervaire-Milnor invariant because these authors first proved Rohlin's formula below [9] for the case where $M$ is smooth and $c$ is represented by an embedded sphere, implying the properties $\tau(c)=0=K S(M)$.

Remark 3. The figure above shows a framed Whitney disk $W_{i}$ in the sense that there are two disjoint parallel copies of $W_{i}$, as needed for the Whitney move on the right hand side. In general, a Whitney disk comes with a framing of its boundary and hence admits a well defined Euler number in $\mathbb{Z}$, its twist. The operation of boundary twisting [7] allows to assume that all Whitney disks are framed, i.e. have twist zero. Moreover, one can also assume that the $W_{i}$ are (disjointly) embedded disks, by pushing all (self)-intersections off the boundary.

A generalization of Rohlin's theorem [6] says that this geometric invariant determines the Kirby-Siebenmann invariant of a closed oriented $4-$ manifold $M$ by the formula

$$
K S(M) \equiv \tau_{M}(c)+\frac{\lambda_{M}(c, c)-\sigma\left(\lambda_{M}\right)}{8} \bmod 2
$$

explaining the equivalence of criteria (iv) and (v) above. In Section 2 we'll recall a definition of $\tau_{M}$ which makes the above formula hold for all closed oriented 4 -manifolds $M$ (without assuming that $c$ is spherical).

The 2-complex $\mathcal{W}:=S \cup W_{i}$ in $M$ is referred to as a Whitney tower of order 1 , built on $S$, with order 1 Whitney disks $W_{i}$. The invariant $\tau_{1}(\mathcal{W})=\tau_{1}\left(S, W_{i}\right)$ used above is the first intersection invariant of such Whitney towers. It has again order 1, the order zero 
intersection invariants being given by the intersection form $\lambda_{M}$. In a sequence of papers, the authors generalized this invariant to higher orders, see for example our survey [3].

The idea is that if $\tau_{1}(\mathcal{W})$ vanishes then all intersections between $S$ and $W_{i}$ can be paired by order 2 Whitney disks $W_{i, j}$ and there should be a second-order intersection invariant $\tau_{2}\left(\mathcal{W}, W_{i, j}\right)$ measuring the obstruction for finding order 3 Whitney disks, and so on.

In [4] we worked out this higher-order intersection theory in detail for Whitney towers built on immersed disks in the 4-ball bounded by framed links in the 3-sphere. In this simply connected setting the invariant $\tau_{n}(\mathcal{W})$ of an order $n$ (framed) Whitney tower $\mathcal{W}$ takes values in an abelian group $\mathcal{T}_{k}(m)$ (where $m$ is number of link components), and the vanishing of $\tau_{n}(\mathcal{W})$ implies that the link bounds and order $n+1$ Whitney tower. For links bounding twisted Whitney towers there is an analogous obstruction theory and intersection invariant $\tau_{k}^{\infty}(\mathcal{W}) \in \mathcal{T}_{k}^{\infty}(m)$, and in the second part of this paper we develop an algebraic theory of quadratic forms, leading to a beautiful relation between these framed and twisted obstruction groups, spelled out in Theorem 9. This result is used in the computation of the Whitney tower filtration on classical links described in [4].

Acknowledgments: The main part of this paper was written while the first two authors were visiting the third author at the Max-Planck-Institut für Mathematik in Bonn. They all thank MPIM for its stimulating research environment and generous support. The third author was also supported by NSF grants DMS-0806052 and DMS-0757312.

\section{Combinatorial approach to the Kirby-Siebenmann invariant}

In the notation of the introduction, recall that a class $c \in \mathbb{Z}^{m}$ is called characteristic if

$$
\lambda(c, x) \equiv \lambda(x, x) \quad \bmod 2 \quad \forall x \in \mathbb{Z}^{m}
$$

The set $C(\lambda)$ of characteristic elements is a $\mathbb{Z}^{m}$-torsor via the action $(c, x) \mapsto c+2 x$.

A closed oriented 4-manifold $M$ with $\left(H_{2} M, \lambda_{M}\right)=\left(\mathbb{Z}^{m}, \lambda\right)$ defines a quadratic refinement $\tau_{M}: C(\lambda) \rightarrow \mathbb{Z}_{2}$ of $\lambda$ in the sense that

$$
\tau_{M}(c+2 x) \equiv \frac{\lambda(c, x)-\lambda(x, x)}{2} \bmod 2
$$

This means that $\tau_{M}$ is completely determined by one of its values (and that value is determined by the Kirby-Siebenmann invariant of $M$ via Rohlin's theorem above). If $\lambda$ is even then $c=0$ is characteristic and a simple geometric argument shows that $\tau_{M}(0)=0$, see below. That's why $\tau_{M}$ is only interesting for odd intersection forms.

Freedman and Kirby [6, 10] defined $\tau_{M}(c)$ by representing $c$ by an embedded surface $\Sigma \subset M$ such that $M \backslash \Sigma$ has a spin structure that does not extend across $\Sigma$. Let $\pi: S \nu(\Sigma, M) \rightarrow \Sigma$ be the projection map of the boundary of a normal disk bundle for $\Sigma$. Then there is a quadratic refinement $q$ of the intersection form on $H_{1}(\Sigma)$ defined by taking the inverse image torus $\pi^{-1}(a)$ for a circle $a$ in $\Sigma$ and observing that it comes equipped with a canonical spin structure. Then $q(a) \in \mathbb{Z}_{2}$ is the Arf invariant of this spin torus, aka its spin bordism class, and finally $\tau_{M}(c)$ is the Arf invariant of $q$.

This definition can be drastically simplified if $c$ is represented by an immersed sphere $S$ whose self-intersection points are paired by framed Whitney disks $W_{1}, \ldots, W_{g}$. (This is always the case if $M$ is simply-connected as in the introduction.)

Lemma 4. Define $\tau_{1}\left(S, W_{i}\right) \in \mathbb{Z}_{2}$ to be the sum of all intersections between the immersed sphere $S$ and the interiors of the Whitney disks $W_{i}$. Then $\tau_{1}\left(S, W_{i}\right)=\tau_{M}(c)$. 
Proof. In [6] the following simplification of $\tau_{M}(c)$ is already explained, in fact, this was the original definition (and only later it was realized that one needs a more general approach because caps don't always exist): Assume that $c$ is represented by a surface $\Sigma$ with (immersed, framed) caps. These are (immersed, framed) disks $A_{1}, \ldots, A_{g}, B_{1}, \ldots, B_{g}$ in $M$ bounding a hyperbolic basis $a_{1}, \ldots, a_{g}, b_{1}, \ldots, b_{g}$ of curves in $H_{1}(\Sigma)$.

Freedman-Kirby show that the quadratic form $q: H_{1}(\Sigma) \rightarrow \mathbb{Z}_{2}$ is determined by $q\left(a_{i}\right)=$ number of intersections between the interior of the cap $A_{i}$ and $\Sigma$, similarly for $q\left(b_{i}\right)$. By definition of the Arf invariant, one gets that

$$
\tau_{M}(c)=\sum_{i=1}^{g} q\left(a_{i}\right) \cdot q\left(b_{i}\right)
$$

Assume now that $c$ is represented by an immersed sphere $S$ whose self-intersection points are paired by (immersed, framed) Whitney disks $W_{1}, \ldots, W_{k}$. We can get into the capped surface situation as follows: For each pair of self-intersection points of $S$, add a tube $T_{i}$ on one sheet going from one self-intersection to the other. That turns $S$ into an embedded surface $\Sigma$ with half of the caps $A_{i}$ given by small normal disks to $\Sigma$ that bound the generating circles on $T_{i}$. Moreover, the Whitney disks $W_{i}$ can serve as the dual caps $B_{i}$, preserving the framing, as illustrated in Figure 2 .

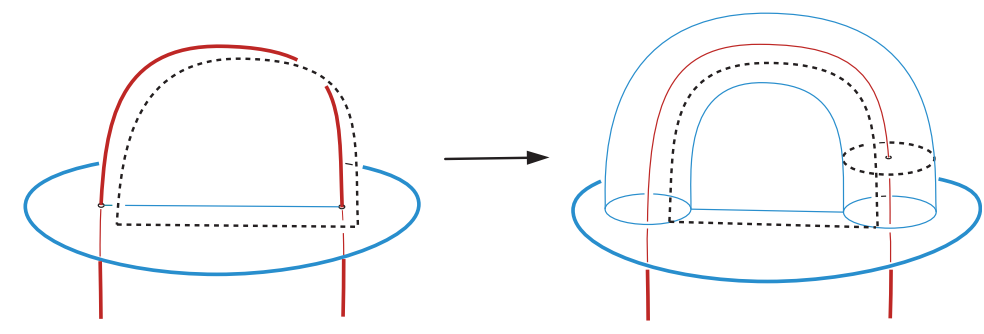

FiguRE 2. Turning an immersed sphere with Whitney disks into a capped surface.

By construction, $q\left(a_{i}\right)=1$ since each normal disk $A_{i}$ intersects $\Sigma$ in a single point. Therefore, the required formula follows:

$$
\tau_{M}(c)=\sum_{i=1}^{g} q\left(b_{i}\right)=\tau_{1}\left(S, W_{i}\right)
$$

Remark 5. In the simply-connected case, it is not hard to see that $\tau_{1} \in \mathbb{Z}_{2}$ is well-defined exactly on characteristic elements. One thing to check is that it does not depend on the choices of the Whitney disks $W_{i}$. Once we fix the boundary, any two such choices differ by a connected sum into a sphere $S_{i}$. If we require the Whitney disks to be (stably) framed then $S_{i}$ needs to be (stably) framed and hence it intersects a characteristic sphere in an even number of points, leaving our count $\tau_{1}$ unchanged modulo two.

All these considerations can be found in chapter 10 of the book [7] by Freedman and Quinn. Unfortunately, the results don't hold as stated for 4-manifolds with fundamental groups that contain 2-torsion elements. The problem arises from different choices of pairings of intersection points, as observed by Richard Stong in [13]. In [12], the last two authors gave a complete discussion of the invariant $\tau_{1}$ in the presence of fundamental groups. 


\section{Abelian groups generated By trees}

All trees considered in this paper are unitrivalent, oriented and labelled. This means that they are equipped with vertex orientations, i.e. cyclic orderings of the edges incident to each trivalent vertex. Moreover, the univalent vertices of a tree are labeled by elements of the index set $\{1,2, \ldots, m\}$, except for the unlabeled root vertex if a given tree happens to be rooted. (There is at most one root, even though any other index can appear several times). All trees are considered up to label-preserving isomorphism.

The order of such a tree is the number of trivalent vertices.
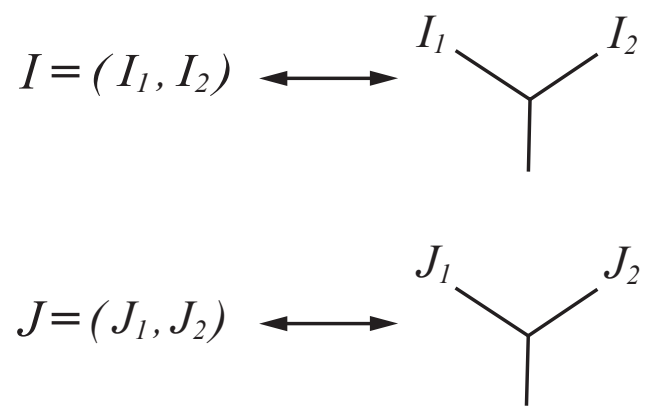
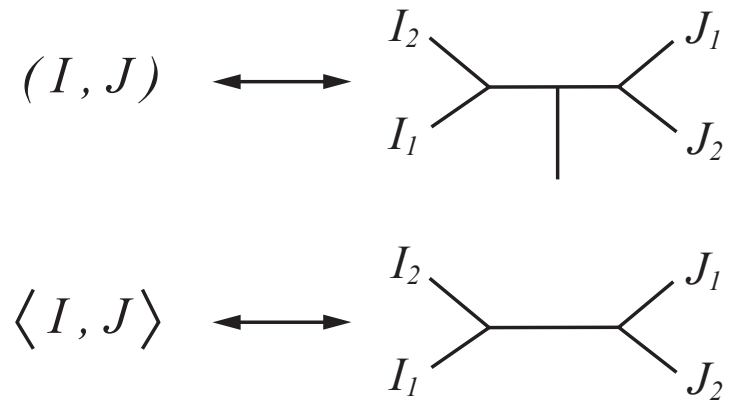

FiguRE 3. Rooted and inner products.

Given rooted trees $I$ and $J$, the rooted product $(I, J)$ is the rooted tree gotten by identifying the two roots to a vertex and adjoining a rooted edge to this new vertex, with the orientation of the new trivalent vertex given by the ordering of $I$ and $J$ in $(I, J)$. The inner product $\langle I, J\rangle$ of two rooted trees $I$ and $J$ is defined to be the unrooted tree gotten by identifying the two rooted edges to a single edge. We observe that the two products interact well in the sense of Figure 4 .

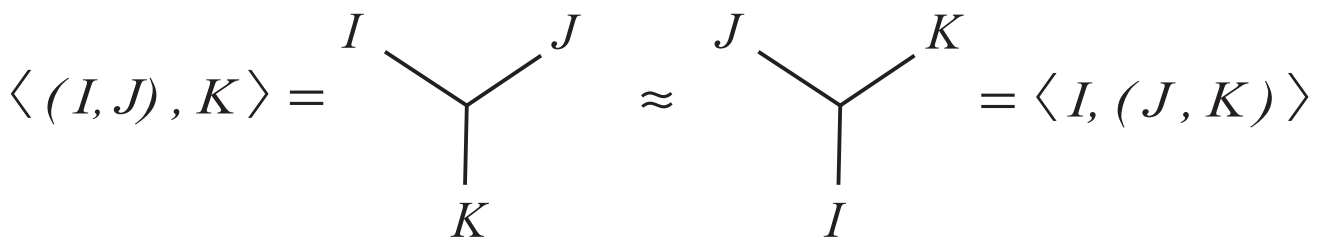

FiguRE 4. Invariance of the inner product.

Let $\mathbb{L}(m)$ be the free abelian group generated by (isomorphism classes of) rooted trees as above. It is graded by order and the rooted product can be extended linearly to a pairing

$$
(,): \mathbb{L}(m) \otimes \mathbb{L}(m) \longrightarrow \mathbb{L}(m)
$$

This is grading preserving on $\mathbb{L}(m)[1]$, i.e. it preserves the grading when shifted up by one (so order is replaced by the number of univalent non-root vertices). On the other hand, the inner product

$$
\langle,\rangle: \mathbb{L}(m) \otimes \underset{5}{\mathbb{L}}(m) \longrightarrow \mathbb{T}(m)
$$


is grading preserving via order. Here $\mathbb{T}(m)$ is the free abelian group generated by unrooted trees as above.

Note that rotating the relevant planar trees by 180 respectively 120 degrees shows that the inner product is both symmetric and invariant: $\langle I, J\rangle=\langle J, I\rangle$ and $\langle(I, J), K\rangle=\langle I,(J, K)\rangle$, see Figure 4 for the proof of invariance.

Definition 6. The graded abelian groups $\mathcal{L}(m)$ respectively $\mathcal{T}(m)$ are defined as quotients of $\mathbb{L}(m)$ respectively $\mathbb{T}(m)$ by the AS and IHX relations as in Figure 5 .

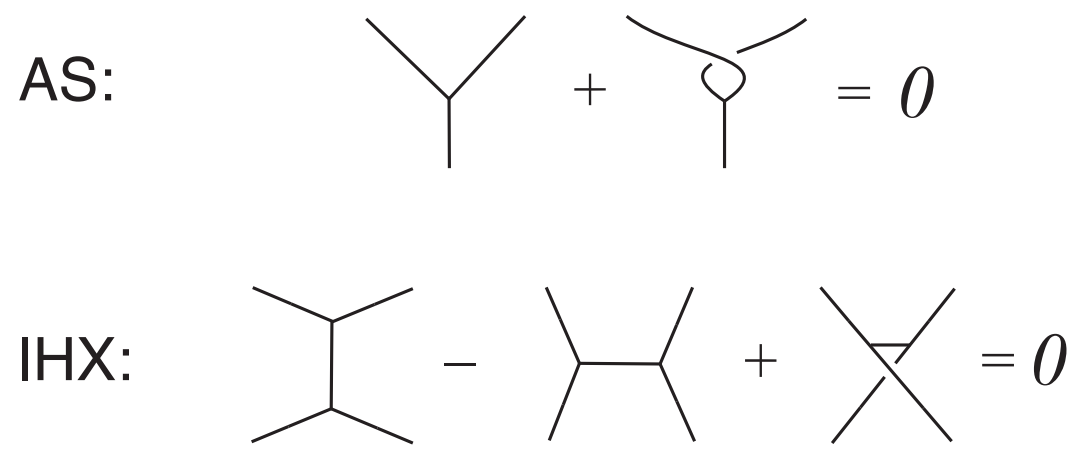

FiguRE 5. Local antisymmetry (AS) and Jacobi (IHX) relations in $\mathcal{L}(m)$ and $\mathcal{T}(m)$. All trivalent orientations come from an orientation of the plane, and univalent vertices extend to subtrees which are fixed in each equation.

It is well known that $\mathcal{L}(m)$ is the free (quasi) Lie algebra over $\mathbb{Z}$ on $m$ generators with Lie bracket induced by the rooted product. Here the word quasi refers to the fact that we only require the antisymmetry relations $[X, Y]=-[Y, X]$. So $[X, X]$ is not necessarily zero in these Lie algebras. In our previous papers, we needed to consider both versions of Lie algebras and used the notation $\mathrm{L}_{n+1}^{\prime}(m)$ for $\mathcal{L}_{n}(m)$ (recall that one gets a graded Lie algebra only when shifting the order by one). In this paper we will only study one type of Lie algebras and usually omit the adjective 'quasi'.

Remark 7. The inner product extends uniquely to a bilinear, symmetric, invariant pairing

$$
\langle,\rangle: \mathcal{L}(m) \times \mathcal{L}(m) \longrightarrow \mathcal{T}(m)
$$

This follows simply from observing that the AS and IHX relations hold on both sides and are preserved by the inner product. We will show in Lemma 10 that this inner product is in fact universal.

Definition 8. The group $\mathcal{T}_{2 n}^{\infty}(m)$ is gotten from $\mathcal{T}_{2 n}(m)$ by adding order $n$ cs-trees as new generators. These are rooted trees of order $n$ as above, except that the root carries the label œ. In addition to the IHX- and AS-relations on unrooted trees in $\mathcal{T}_{2 n}(m)$, we introduce the following new symmetry, interior twist and twisted IHX relations:

$$
J^{\infty}=(-J)^{\infty} \quad 2 \cdot J^{\infty}=\langle J, J\rangle \quad I^{\infty}=H^{\infty}+X^{\infty}-\langle H, X\rangle
$$

As their names suggest, these new relations arose from geometric considerations for twisted Whitney towers in [4]. They will be explained algebraically in our last section via the theory of universal quadratic refinements. 
Roughly speaking, the universal symmetric pairing $\langle$,$\rangle will be shown to admit a universal$ quadratic refinement $q: \mathcal{L}_{n}(m) \rightarrow \mathcal{T}_{2 n}^{\infty}(m)$ defined by $q(J):=J^{\infty}$. In particular, with the right algebraic notion of 'quadratic refinement', the group $\mathcal{T}_{2 n}^{\infty}(m)$ is completely determined by the pairing $\langle$,$\rangle . The rest of this paper is devoted to finding this notion.$

As a consequence, we will prove the following exact sequence at the very end of this paper. It was used substantially in [4] for the classification of Whitney towers in the 4-ball.

Theorem 9. For all $m, n$, the maps $t \mapsto t$ respectively $J^{\infty} \mapsto 1 \otimes J$ give an exact sequence:

$$
0 \longrightarrow \mathcal{T}_{2 n}(m) \longrightarrow \mathcal{T}_{2 n}^{\infty}(m) \longrightarrow \mathbb{Z}_{2} \otimes \mathcal{L}_{n}(m) \longrightarrow 0
$$

\section{INVARIANT FORMS AND QUADRATIC REFINEMENTS}

In this section we explain an algebraic framework into which our groups $\mathcal{T}(m)$ and $\mathcal{T}^{\infty}(m)$ fit naturally. In Lemma 10 we show that the $\mathcal{T}(m)$-valued inner product $\langle\cdot, \cdot\rangle$ on the free Lie algebra is universal. Then a general theory of quadratic refinements is developed and specialized from the non-commutative to the commutative to finally, symmetric settings. In Corollary 33 we show that $\mathcal{T}_{2 n}^{\infty}(m)$ is the home for the universal quadratic refinement of the $\mathcal{T}_{2 n}(m)$-valued inner product $\langle$,$\rangle .$

We work over the ground ring of integers but all our arguments go through for any commutative ring. We also only discuss the case of finite generating sets $\{1, \ldots, m\}$, even though everything holds in the infinite case.

4.1. A universal invariant form. The following lemma shows that this inner product is universal for Lie algebras with $m$ generators.

Lemma 10. Let $\mathfrak{g}$ be a Lie algebra together with a bilinear, symmetric, invariant pairing $\lambda: \mathfrak{g} \times \mathfrak{g} \rightarrow M$ into some abelian group $M$. If $\alpha: \mathcal{L}(m) \rightarrow \mathfrak{g}$ is a Lie homomorphism (given by $m$ arbitrary elements in $\mathfrak{g})$ there exists a unique linear map $\Psi: \mathcal{T}(m) \rightarrow M$ such that for all $X, Y \in \mathcal{L}(m)$

$$
\lambda(\alpha(X), \alpha(Y))=\Psi(\langle X, Y\rangle)
$$

Proof. The uniqueness of $\Psi$ is clear since the inner product map is onto. For existence, we first construct a map $\psi: \mathbb{T}(m) \rightarrow M$ as follows. Given a tree $t \in \mathbb{T}(m)$ pick an edge in $t$ to split $t=\langle X, Y\rangle$ for rooted trees $X, Y \in \mathbb{L}(m)$. Then set

$$
\psi(t):=\lambda(\alpha(X), \alpha(Y))
$$

If we split $t$ at an adjacent edge, this expression stays unchanged because of the symmetry and invariance of $\lambda$. However, one can go from any given edge to any other by a sequence of adjacent edges, showing that $\psi(t)$ does not depend on the choice of splitting.

It is clear that $\psi$ can be extended linearly to the free abelian group on $\mathbb{T}(m)$ and since $\alpha$ preserves AS and IHX relations by assumption, this extension factors through a map $\Psi$ as required.

Remark 11. Recall that $\mathcal{L}(m)[1]$ is actually a graded Lie algebra, i.e. the Lie bracket preserves the grading when shifted up by one (so order is replaced by the number of univalent non-root vertices). Let's assume in the above lemma that the groups $\mathfrak{g}, M$ are $\mathbb{Z}$-graded, $\mathfrak{g}[1]$ is a graded Lie algebra and that $\lambda, \alpha$ preserve those gradings. Then the proof shows that the resulting linear map $\Psi$ also preserves the grading. 
4.2. Non-commutative quadratic groups. The rest of this section describes a general setting for relating our groups $\mathcal{T}_{2 n}^{\infty}(m)$ that measure the intersection invariant of twisted Whitney towers to a universal (symmetric) quadratic refinement of the $\mathcal{T}_{2 n}(m)$-valued inner product. We first give a couple of definitions that generalize those introduced by Hans Baues in [1] and [2, §8], and Ranicki in [11, p.246]. These will lead to the most general notion of quadratic refinements for which we construct a universal example. Later we shall specialize the definitions from non-commutative to commutative and finally, to symmetric quadratic forms and construct universal examples in all cases.

Definition 12. A (non-commutative) quadratic group

$$
\mathfrak{M}=\left(M_{e} \stackrel{h}{\rightarrow} M_{e e} \stackrel{p}{\rightarrow} M_{e}\right)
$$

consists of two groups $M_{e}, M_{e e}$ and two homomorphisms $h, p$ satisfying

(i) $M_{e e}$ is abelian,

(ii) the image of $p$ lies in the center of $M_{e}$,

(iii) $h p h=2 h$.

$\mathfrak{M}$ will serve as the range of the (non-commutative) quadratic forms defined below. We will write both groups additively since in most examples $M_{e}$ turns out to be commutative. A morphism $\beta: \mathfrak{M} \rightarrow \mathfrak{M}^{\prime}$ between quadratic groups is a pair of homomorphisms

$$
\beta_{e}: M_{e} \rightarrow M_{e}^{\prime} \quad \text { and } \quad \beta_{e e}: M_{e e} \rightarrow M_{e e}^{\prime}
$$

such that both diagrams involving $h, h^{\prime}, p, p^{\prime}$ commute.

Examples 13. The example motivating the notation comes from homotopy theory, see e.g. [1]. For $m<3 n-2$, let $M_{e}=\pi_{m}\left(S^{n}\right), M_{e e}=\pi_{m}\left(S^{2 n-1}\right), h$ be the Hopf invariant and $p$ be given by post-composing with the Whitehead product $\left[\iota_{n}, \iota_{n}\right]: S^{2 n-1} \rightarrow S^{n}$.

This quadratic group satisfies $p h p=2 p$ which is part of the definition used in [1], where $M_{e}$ is also assumed to be commutative. As we shall see, these additional assumptions have the disadvantage that they are not satisfied for the universal example 18 .

Another important example comes from an abelian group with involution $(M, *)$. Then we let

$$
M_{e e}:=M, \quad M_{e}:=M /\left\langle x-x^{*}\right\rangle, \quad h([x]):=x+x^{*}
$$

and $p$ be the natural quotient map. For example, if $M$ is a ring with involution $r \mapsto \bar{r}$, then we get two possible involutions on the abelian group $M: r^{*}= \pm \bar{r}$. The choice of sign determines whether we study symmetric respectively skew-symmetric pairings.

We note that in this example $h p-\mathrm{id}=*$ and in the homotopy theoretic example $h p-\mathrm{id}=$ $(-1)^{n}$. In fact, we have the following

Lemma 14. Given a quadratic group, the endomorphism hp - id gives an involution on $M_{e e}$ (which we will denote by $*$ ). Moreover, the formula $\dagger(x):=p h(x)-x$ defines an anti-involution on $M_{e}$. These satisfy

(i) $* \circ h=h$,

(ii) $p h p=p+p \circ *$,

(iii) $p \circ *=\dagger \circ p$. 
The proof of Lemma 14 is straightforward and will be left to the reader. To show that $\dagger$ is an anti-homomorphism one uses that $\operatorname{Im}(p)$ is central and that $x \mapsto-x$ is an antihomomorphism.

Definition 15. A quadratic group $\mathfrak{M}$ is a quadratic refinement of an abelian group with involution $(M, *)$ if

$$
M_{e e}=M \quad \text { and } \quad *=h p-\mathrm{id} .
$$

It follows from (i) in Lemma 14 that in this case, the image of $h$ lies in the fixed point set of the involution: $h: M_{e} \rightarrow M^{\mathbb{Z} 2}=H^{0}\left(\mathbb{Z}_{2} ; M\right)$.

The example $(M)$ above gives one natural choice of a quadratic refinement, however, there are other canonical (and non-commutative) ones as we shall see in Example 18.

It follows from (ii) in Lemma 14 that the additional condition $p h p=2 p$ used in [1] is satisfied if and only if $p=p \circ *$, or equivalently, if $p$ factors through the cofixed point set of the involution:

$$
p: M_{e e} \rightarrow\left(M_{e e}\right)_{\mathbb{Z}_{2}}=H_{0}\left(\mathbb{Z}_{2} ; M_{e e}\right) \rightarrow M_{e}
$$

It follows that the notion in [11, p.246] is equivalent to that in [1], except that $M_{e e}$ is assumed to be the ground ring $R$ in the former. In that case, our involution is simply $r^{*}=\epsilon \bar{r}$, where $\epsilon= \pm 1$ and $r \mapsto \bar{r}$ is the given involution on the ring $R$.

Then $\epsilon$-symmetric forms in the sense of Ranicki become hermitian forms in the sense defined below. In particular, Ranicki's $(+1)$-symmetric forms are different from the notion of symmetric form in this paper: We reserve it for the easiest case where both involutions, $*$ and $\dagger$, are trivial.

\subsection{Non-commutative quadratic forms.}

Definition 16. A (non-commutative) quadratic form on an abelian group $A$ with values in a (non-commutative) quadratic group $\mathfrak{M}=\left(M_{e} \stackrel{h}{\rightarrow} M_{e e} \stackrel{p}{\rightarrow} M_{e}\right)$ is given by a bilinear map $\lambda: A \times A \rightarrow M_{e e}$ and a map $\mu: A \rightarrow M_{e}$ satisfying

(i) $\mu\left(a+a^{\prime}\right)=\mu(a)+\mu\left(a^{\prime}\right)+p \circ \lambda\left(a, a^{\prime}\right)$ and

(ii) $h \circ \mu(a)=\lambda(a, a) \forall a, a^{\prime} \in A$.

We say that $\mu$ is a quadratic refinement of $\lambda$ : Property (i) says that $\mu$ is quadratic and property (ii) means that it "refines" $\lambda$. The notation $M_{e}$ and $M_{e e}$ was designed (by Baues) to reflect the number of variables (entries) of the maps $\mu$ and $\lambda$ respectively. He also writes $\lambda=\lambda_{e e}$ and $\mu=\lambda_{e}$, however, we decided not to follow that part of the notation.

We write $(\lambda, \mu): A \rightarrow \mathfrak{M}$ for such quadratic forms and we always assume that the quadratic group $\mathfrak{M}$ is part of the data for $(\lambda, \mu)$. This means that the morphisms in the category of quadratic forms are pairs of morphisms

$$
\alpha: A \rightarrow A^{\prime} \quad \text { and } \quad \beta=\left(\beta_{e}, \beta_{e e}\right): \mathfrak{M} \rightarrow \mathfrak{M}^{\prime}
$$

such that both diagrams involving $\lambda, \lambda^{\prime}, \mu, \mu^{\prime}$ commute.

Lemma 17. Let $(\lambda, \mu): A \rightarrow \mathfrak{M}$ be a quadratic form as above. Then $\lambda$ is hermitian with respect to the involution $*=h p-\mathrm{id}$ on $M_{e e}$ :

$$
\lambda\left(a^{\prime}, a\right)=\lambda\left(a, a^{\prime}\right)^{*}
$$

and $\mu$ is hermitian with respect to the anti-involution $\dagger=$ ph $-\mathrm{id}$ on $M_{e}$ :

$$
\mu(-a)=\mu(a)^{\dagger}
$$


Proof. As a consequence of conditions (i) and (ii) we get

$$
\begin{aligned}
& \lambda(a, a)+\lambda\left(a^{\prime}, a^{\prime}\right)+\lambda\left(a^{\prime}, a\right)+\lambda\left(a, a^{\prime}\right)=\lambda\left(a+a^{\prime}, a+a^{\prime}\right)= \\
& h \circ \mu\left(a+a^{\prime}\right)=h\left(\mu(a)+\mu\left(a^{\prime}\right)+p \circ \lambda\left(a, a^{\prime}\right)\right)= \\
& \lambda(a, a)+\lambda\left(a^{\prime}, a^{\prime}\right)+h p\left(\lambda\left(a, a^{\prime}\right)\right)
\end{aligned}
$$

or equivalently, $\lambda\left(a^{\prime}, a\right)=(h p-\mathrm{id}) \lambda\left(a, a^{\prime}\right)=\lambda\left(a, a^{\prime}\right)^{*}$. Similarly,

$$
\begin{aligned}
& 0=\mu(0)=\mu(a-a)=\mu(a)+\mu(-a)+p \circ \lambda(a,-a)= \\
& \mu(a)+\mu(-a)-p \circ h \circ \mu(a)=\mu(-a)+(\mathrm{id}-p h) \mu(a)
\end{aligned}
$$

or equivalently, $\mu(-a)=\dagger \circ \mu(a)=: \mu(a)^{\dagger}$.

Starting with a hermitian form $\lambda$ with values in a group with involution $(M, *)$, the first step in finding a quadratic refinement for $\lambda$ is to find a quadratic refinement $\mathfrak{M}$ of $(M, *)$ in the sense of Definition 15, motivating our terminology.

\subsection{Universal quadratic refinements.}

Example 18. Given a hermitian form $\lambda: A \times A \rightarrow(M, *)$, one gets a quadratic refinement $\mu_{\lambda}$ of $\lambda$ as follows. Set $M_{e e}:=M$ and define the universal target $M_{e}:=M_{e e} \times_{\lambda} A$ to be the group consisting of pairs $(m, a)$ with $m \in M_{e e}$ and $a \in A$ and multiplication given by

$$
(m, a)+\left(m^{\prime}, a^{\prime}\right):=\left(m+m^{\prime}-\lambda\left(a, a^{\prime}\right), a+a^{\prime}\right)
$$

In other words, $M_{e}$ is the central extension

$$
1 \longrightarrow M_{e e} \longrightarrow M_{e e} \times_{\lambda} A \longrightarrow A \longrightarrow 1
$$

determined by the cocycle $\lambda$, compare Section 4.7. It follows that $M_{e}$ is commutative if and only if $\lambda$ is symmetric in the naive sense that $\lambda\left(a^{\prime}, a\right)=\lambda\left(a, a^{\prime}\right)$. Set

$$
p_{\lambda}(m):=(m, 0), \quad h_{\lambda}(m, a):=m+m^{*}+\lambda(a, a)
$$

We claim that $\mathfrak{M}_{\lambda}:=\left(M_{e e} \stackrel{p_{\lambda}}{\rightarrow} M_{e} \stackrel{h_{\lambda}}{\rightarrow} M_{e e}\right)$ is a quadratic group as in Definition 12 . It is clear that $p_{\lambda}$ is a homomorphism with image in the center of $M_{e}$. The homomorphism property of $h_{\lambda}$ follows from the fact that $\lambda$ is bilinear and hermitian:

$$
\begin{aligned}
& h_{\lambda}\left((m, a)+\left(m^{\prime}, a^{\prime}\right)\right)=h_{\lambda}\left(m+m^{\prime}-\lambda\left(a, a^{\prime}\right), a+a^{\prime}\right)= \\
& \left(m+m^{\prime}-\lambda\left(a, a^{\prime}\right)\right)+\left(m+m^{\prime}-\lambda\left(a, a^{\prime}\right)\right)^{*}+\lambda\left(a+a^{\prime}, a+a^{\prime}\right)= \\
& \left(m+m^{*}+\lambda(a, a)\right)+\left(m^{\prime}+m^{*}+\lambda\left(a^{\prime}, a^{\prime}\right)\right)=h_{\lambda}(m, a)+h_{\lambda}\left(m^{\prime}, a^{\prime}\right)
\end{aligned}
$$

Condition (iii) of a quadratic group is also checked easily:

$$
\begin{aligned}
& h_{\lambda} p_{\lambda} h_{\lambda}(m, a)=h_{\lambda}\left(m+m^{*}+\lambda(a, a), 0\right)= \\
& \left(m+m^{*}+\lambda(a, a)\right)+\left(m+m^{*}+\lambda(a, a)\right)^{*}= \\
& 2\left(m+m^{*}+\lambda(a, a)\right)=2 h_{\lambda}(m, a)
\end{aligned}
$$

We also see that

$$
\left(h_{\lambda} p_{\lambda}-\mathrm{id}\right)(m)=h_{\lambda}(m, 0)-m=\left(m+m^{*}\right)-m=m^{*}
$$

which means that $\mathfrak{M}_{\lambda}$ "refines" (in the sense of Definition 15) the group with involution $(M, *)$. Finally, setting $\mu_{\lambda}(a):=(0, a)$, we claim that $\left(\lambda, \mu_{\lambda}\right): A \rightarrow \mathfrak{M}_{\lambda}$ is a quadratic refinement of $\lambda$. We need to check properties (i) and (ii) of a quadratic form (Definition 16): 
(i) is simply $h_{\lambda} \circ \mu_{\lambda}(a)=h_{\lambda}(0, a)=\lambda(a, a)$, and (ii) explains why we used a sign in front of $\lambda$ in our central extension:

$$
\begin{aligned}
& \mu_{\lambda}(a)+\mu_{\lambda}\left(a^{\prime}\right)+p_{\lambda} \circ \lambda\left(a, a^{\prime}\right)=(0, a)+\left(0, a^{\prime}\right)+\left(\lambda\left(a, a^{\prime}\right), 0\right)= \\
& \left(-\lambda\left(a, a^{\prime}\right), a+a^{\prime}\right)+\left(\lambda\left(a, a^{\prime}\right), 0\right)=\left(0, a+a^{\prime}\right)=\mu_{\lambda}\left(a+a^{\prime}\right)
\end{aligned}
$$

The following result will show that $\mu_{\lambda}$ is indeed a universal quadratic refinement of $\lambda$. This is the content of the first statement in the theorem below. It follows from the second statement because for any quadratic refinement $\mu$ of $\lambda$ it shows that forgetting the quadratic data gives canonical isomorphisms

$$
\mathrm{QF}(L \circ R(\lambda, \mu),(\lambda, \mu)) \cong \mathrm{HF}(R(\lambda, \mu), R(\lambda, \mu))=\mathrm{HF}(\lambda, \lambda)
$$

where QF respectively HF are (the morphisms in) the categories of quadratic respectively hermitian forms. Since

$$
L \circ R(\lambda, \mu)=L(\lambda)=\left(\lambda, \mu_{\lambda}\right)
$$

and the morphisms in the category $\mathrm{QR}_{\lambda}$ of quadratic refinements of $\lambda$ by definition all lie over the identity of $\lambda$, the set $\mathrm{QR}_{\lambda}\left(\mu_{\lambda}, \mu\right)$ contains a unique element, namely the required universal morphism $\mu_{\lambda} \rightarrow \mu$.

Theorem 19. The quadratic form $\left(\lambda, \mu_{\lambda}\right)$ is initial in the category of quadratic refinements of $\lambda$. In fact, the forgetful functor $R(\lambda, \mu)=\lambda$ from the category of quadratic forms to the category of hermitian forms has a left adjoint $L: \mathrm{HF} \rightarrow \mathrm{QF}$ given by $L(\lambda):=\left(\lambda, \mu_{\lambda}\right)$.

Proof. We have to construct natural isomorphisms

$$
\mathrm{QF}\left(\left(\lambda, \mu_{\lambda}\right),\left(\lambda^{\prime}, \mu^{\prime}\right)\right)=\mathrm{QF}\left(L(\lambda),\left(\lambda^{\prime}, \mu^{\prime}\right)\right) \cong \operatorname{HF}\left(\lambda, R\left(\lambda^{\prime}, \mu^{\prime}\right)\right)=\operatorname{HF}\left(\lambda, \lambda^{\prime}\right)
$$

for any quadratic form $\left(\lambda^{\prime}, \mu^{\prime}\right)$ and hermitian form $\lambda$. Recall that the morphisms in QF are pairs $\alpha: A \rightarrow A^{\prime}$ and $\beta=\left(\beta_{e}, \beta_{e e}\right): \mathfrak{M} \rightarrow \mathfrak{M}^{\prime}$ such that the relevant diagrams commute. This implies that forgetting about the quadratic datum $\beta_{e}$ gives a natural map from the left to the right hand side above.

Given a morphism $\left(\alpha, \beta_{e e}\right): \lambda \rightarrow \lambda^{\prime}$ consisting of homomorphisms $\alpha: A \rightarrow A^{\prime}$ and $\beta_{e e}:\left(M_{e e}, *\right) \rightarrow\left(M_{e e}^{\prime}, *^{\prime}\right)$ such that

$$
\lambda^{\prime}\left(\alpha\left(a_{1}\right), \alpha\left(a_{2}\right)\right)=\beta_{e e} \circ \lambda\left(a_{1}, a_{2}\right) \in M_{e e}^{\prime} \quad \forall a_{i} \in A
$$

we need to show that there is a unique homomorphism $\beta_{e}: M_{e} \rightarrow M_{e}^{\prime}$ such that the following 3 diagrams commute:
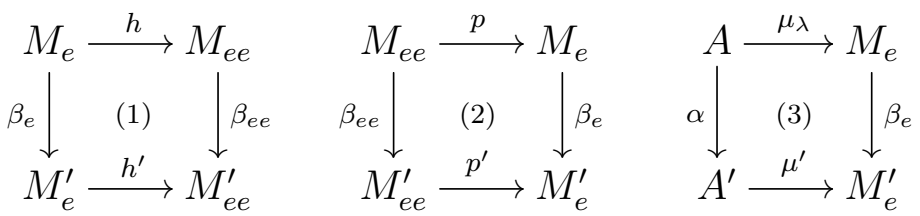

We will now make use of the fact that $M_{e}=M_{e e} \times_{\lambda} A$ because $\mu_{\lambda}$ is given as in Example 18 In this case, diagrams (2) and (3) are equivalent to

$$
\beta_{e}(m, 0)=p^{\prime} \circ \beta_{e e}(m) \quad \text { and } \quad \beta_{e}(0, a)=\mu^{\prime} \circ \alpha(a)
$$

because $p(m)=(m, 0)$ and $\mu_{\lambda}(a)=(0, a)$. This implies directly the uniqueness of $\beta_{e}$. For existence, we only have to check that the formula

$$
\beta_{e}(m, a):=p^{\prime} \circ \beta_{e e}(m)+\mu^{\prime} \circ \alpha(a)
$$


gives indeed a group homomorphism $M_{e} \rightarrow M_{e}^{\prime}$ that makes diagram (1) commute. Note that the image of $p^{\prime}$ is central in $M_{e}^{\prime}$ and hence the order of the summands does not matter. We have

$$
\begin{array}{ll}
\beta_{e}\left((m, a)+\left(m^{\prime}, a^{\prime}\right)\right)=\beta_{e}\left(m+m^{\prime}-\lambda\left(a, a^{\prime}\right), a+a^{\prime}\right) & = \\
p^{\prime} \circ \beta_{e e}\left(m+m^{\prime}-\lambda\left(a, a^{\prime}\right)\right)+\mu^{\prime} \circ \alpha\left(a+a^{\prime}\right) & = \\
p^{\prime} \circ \beta_{e e}(m)+p^{\prime} \circ \beta_{e e}\left(m^{\prime}\right)-p^{\prime} \circ \lambda^{\prime}\left(\alpha(a), \alpha\left(a^{\prime}\right)\right)+\mu^{\prime} \circ \alpha\left(a+a^{\prime}\right) & = \\
p^{\prime} \circ \beta_{e e}(m)+p^{\prime} \circ \beta_{e e}\left(m^{\prime}\right)+\mu^{\prime} \circ \alpha(a)+\mu^{\prime} \circ \alpha\left(a^{\prime}\right) & = \\
\beta_{e}(m, a)+\beta_{e}\left(m^{\prime}, a^{\prime}\right) &
\end{array}
$$

To get to the fourth line, we used property (ii) of a quadratic form to cancel the term $p^{\prime} \circ \lambda^{\prime}\left(\alpha(a), \alpha\left(a^{\prime}\right)\right)$. For the commutativity of diagram (1) we use property (i) of a quadratic form, as well as the fact that $\beta_{\text {ee }}$ preserves the involution $*$ :

$$
\begin{aligned}
& h^{\prime} \circ \beta_{e}(m, a)=h^{\prime}\left(p^{\prime} \circ \beta_{e e}(m)+\mu^{\prime} \circ \alpha(a)\right)= \\
& h^{\prime} p^{\prime}\left(\beta_{e e}(m)\right)+\lambda^{\prime}(\alpha(a), \alpha(a))=\beta_{e e}(m)^{*^{\prime}}+\beta_{e e}(m)+\beta_{e e} \circ \lambda(a, a)= \\
& \beta_{e e}\left(m^{*}+m+\lambda(a, a)\right)=\beta_{e e} \circ h(m, a)
\end{aligned}
$$

This finishes the proof of left adjointness of $L: H F \rightarrow Q F$.

If the bilinear form $\lambda$ happens to be symmetric, or more precisely, if it takes values in a group $M_{e e}$ with trivial involution $*$, then the above construction still gives a quadratic refinement $\mu_{\lambda}$. Its target quadratic group $\mathfrak{M}_{\lambda}$ has the properties that $M_{e}$ is abelian and $h_{\lambda} p_{\lambda}=2 \mathrm{id}$. It is not hard to see that our construction above leads to the following result.

Theorem 20. For any symmetric form $\lambda$ one can functorially construct a quadratic form $\left(\lambda, \mu_{\lambda}\right)$ that is initial in the category of quadratic refinements of $\lambda$ with trivial involution $*$. In fact, the forgetful functor $R(\lambda, \mu)=\lambda$ from the category of quadratic forms with trivial involution $*$ to the category of symmetric forms has a left adjoint $L(\lambda)=\left(\lambda, \mu_{\lambda}\right)$.

Remark 21. It follows from the above considerations that a quadratic form $(\lambda, \mu)$ is universal if and only if the homomorphism

$$
M_{e e} \times_{\lambda} A \rightarrow M_{e} \quad \text { given by } \quad(m, a) \mapsto p(m)+\mu(a)
$$

is an isomorphism. This is turn is equivalent to

(i) $p: M_{e e} \rightarrow M_{e}$ is injective and

(ii) $\mu: A \rightarrow M_{e} / \operatorname{Im}(p)$ is an isomorphism.

4.5. Commutative quadratic groups and forms. The case where $*$ is non-trivial but the anti-involution $\dagger$ on $M_{e}$ is trivial is even more interesting. In this case, $\lambda$ is still hermitian with respect to $*$ but one is only interested in quadratic refinements $\mu$ that are symmetric in the sense that $\mu(-a)=\mu(a)$. This case deserves its own definition:

Definition 22. A commutative quadratic group

$$
\mathfrak{M}=\left(M_{e} \stackrel{h}{\rightarrow} M_{e e} \stackrel{p}{\rightarrow} M_{e}\right)
$$

consists of two abelian groups $M_{e}, M_{e e}$ and two homomorphism $h, p$ satisfying $p h=2 \mathrm{id}$. 
In fact, a commutative quadratic group is the same thing as a non-commutative quadratic group with trivial anti-involution $\dagger$. This comes from the fact that the squaring map $x \mapsto 2 x$ is a homomorphism if and only if $M_{e}$ is commutative. Our universal example $\mathfrak{M}_{\lambda}$ is in general not commutative because one gets in this case

$$
\begin{aligned}
& \dagger_{\lambda}(m, a)=p_{\lambda} \circ h_{\lambda}(m, a)-(m, a)=p_{\lambda}\left(m+m^{*}+\lambda(a, a)\right)-(m, a)= \\
& \left(m+m^{*}+\lambda(a, a), 0\right)+(-m-\lambda(a, a),-a)=\left(m^{*},-a\right)
\end{aligned}
$$

However, we shall see in Theorem 25 that we can just divide by these relations $(m, a)=$ $\left(m^{*},-a\right)$ to obtain another universal quadratic refinement of a given hermitian form $\lambda$ but this time with values in a commutative quadratic group. Before we work this out, let us mention the essential example from topology.

Example 23. Consider a manifold $X$ of dimension $2 n$ and let $\mathfrak{M}$ be as in (M) from Example 13 with $M=\mathbb{Z}\left[\pi_{1} X\right]$. In particular, we have $p h-\mathrm{id}=\dagger=\mathrm{id}$ but in general the involution $*$ is non-trivial. On group elements, it is given by

$$
g^{*}:=(-1)^{n} w_{1}(g) g^{-1}
$$

with $w_{1}$ (induced by) the first Stiefel-Whitney class of $X$. Then the equivariant intersection form $\lambda=\lambda_{X}$ on $\pi_{n} X$ is bilinear and hermitian as required. Moreover, the self-intersection invariant $\mu_{X}$ defined by Wall [14] gives a quadratic refinement of $\lambda_{X}$, at least on the subgroup $A$ of elements represented by immersed $n$-spheres with vanishing normal Euler number.

In our main Theorem 25 below, we shall use the following

Lemma 24. If $(\lambda, \mu): A \rightarrow \mathfrak{M}$ is a commutative quadratic form, then $\mu(n \cdot a)=n^{2} \cdot \mu(a)$ for all integers $n \in \mathbb{Z}$.

Here we say that a quadratic form $(\lambda, \mu): A \rightarrow \mathfrak{M}$ is commutative if the target quadratic group $\mathfrak{M}$ is commutative, i.e. if the anti-involution $\dagger$ is trivial (Definition 16).

Proof. Since the involution $\dagger=p h-$ id is trivial by assumption, we already know that $\mu(-a)=\mu(a)$ from Lemma 17. Thus it suffices to prove the claim for positive $n>1$ by induction:

$$
\begin{aligned}
\mu((n+1) \cdot a) & =\mu(n \cdot a)+\mu(a)+p \circ \lambda(n \cdot a, a) \\
& =n^{2} \cdot \mu(a)+\mu(a)+n \cdot p \circ h \circ \mu(a) \\
& =\left(n^{2}+1\right) \cdot \mu(a)+n \cdot 2 \cdot \mu(a)=(n+1)^{2} \cdot \mu(a)
\end{aligned}
$$

Here we again used the fact that $p \circ h=2 \mathrm{id}$.

Theorem 25. Any hermitian bilinear form $\lambda$ has a universal commutative quadratic refinement. In fact, the forgetful functor $R(\lambda, \mu)=\lambda$ from the category CQF of commutative quadratic forms to the category $\mathrm{HF}$ of hermitian forms has a left adjoint $L: \mathrm{HF} \rightarrow \mathrm{CQF}, L(\lambda)=$ $\left(\lambda, \mu_{\lambda}^{c}\right)$.

Proof. As hinted to above, we will force the anti-involution $\dagger$ to be trivial in the universal construction of Theorem 20. This means that we should define the universal (commutative) group $M_{e}^{c}$ as the quotient of our previously used group $M_{e e} \times_{\lambda} A$ by the relations

$$
\begin{aligned}
0 & \left.=\left(m^{*},-a\right)-(m, a)=\left(m^{*},-a\right)+(-m-\lambda(a, a)),-a\right) \\
& =\left(m^{*}-m-2 \lambda(a, a),-2 a\right)
\end{aligned}
$$


By setting $a$ respectively $m$ to zero, these relations imply

$$
\left(m^{*}, 0\right)=(m, 0) \quad \text { and } \quad(-2 \lambda(a, a),-2 a)=0
$$

Vice versa, these two types of equations imply the general ones and hence we see that $M_{e}^{c}$ is the quotient of the centrally extended group

$$
1 \longrightarrow M_{e e} /\left(m^{*}=m\right) \longrightarrow M_{e e} /\left(m^{*}=m\right) \times_{\lambda} A \longrightarrow A \longrightarrow 1
$$

by the relations $(-2 \lambda(a, a),-2 a)=0$. We write elements in $M_{e}^{c}$ as $[m, a]$ with the above relations understood. It then follows that $p_{\lambda}^{c}(m):=[m, 0]$ is a homomorphism $M_{e e} \rightarrow M_{e}^{c}$ (which is in general not any more injective). Moreover, our original formula leads to a homomorphism $h_{\lambda}^{c}: M_{e}^{c} \rightarrow M_{e e}$ given by

$$
h_{\lambda}^{c}[m, a]:=h_{\lambda}(m, a)=m+m^{*}+\lambda(a, a)
$$

To see that this is well defined, observe $h_{\lambda}\left(m^{*}, 0\right)=m+m^{*}=h_{\lambda}(m, 0)$ and

$$
h_{\lambda}(-2 \lambda(a, a),-2 a)=-4 \lambda(a, a)+\lambda(-2 a,-2 a)=0
$$

Finally, we set $\mu_{\lambda}^{c}(a):=[0, a]$ to obtain a commutative quadratic refinement of $\lambda$ which is proven exactly as in Theorem 20.

To show that $\mu_{\lambda}^{c}$ is universal, or more generally, that $L(\lambda):=\left(\mu_{\lambda}^{c}, \lambda\right)$ is a left adjoint of the forgetful functor $R$, we proceed as in the proof of Theorem 20. We are given a morphism $\left(\alpha, \beta_{e e}\right): \lambda \rightarrow \lambda^{\prime}$ consisting of homomorphisms $\alpha: A \rightarrow A^{\prime}$ and $\beta_{e e}:\left(M_{e e}, *\right) \rightarrow\left(M_{e e}^{\prime}, *^{\prime}\right)$ such that

$$
\lambda^{\prime}\left(\alpha\left(a_{1}\right), \alpha\left(a_{2}\right)\right)=\beta_{e e} \circ \lambda\left(a_{1}, a_{2}\right) \in M_{e e}^{\prime} \quad \forall a_{i} \in A .
$$

We need to show that there is a unique homomorphism $\beta_{e}: M_{e}^{c} \rightarrow M_{e}^{\prime}$ such that the three diagrams from the proof of Theorem 20 commute. We can use the same formulas as before, if we check that they vanish on our new relations in $M_{e}^{c}$. For this we'll have to use that the given quadratic group $\mathfrak{M}^{\prime}$ is commutative. Recall the formula

$$
\beta_{e}(m, a)=p^{\prime} \circ \beta_{e e}(m)+\mu^{\prime} \circ \alpha(a)
$$

Splitting our relations into two parts as above, it suffices to show that

$$
p^{\prime} \circ \beta_{e e}\left(m^{*}\right)=p^{\prime} \circ \beta_{e e}(m) \quad \text { and } \quad \beta_{e}(-2 \lambda(a, a),-2 a)=0
$$

The first equation follows from part (iii) of Lemma 14 and the fact that we are assuming that $\dagger^{\prime}=\mathrm{id}$ :

$$
p^{\prime} \circ \beta_{e e}\left(m^{*}\right)=\left(p^{\prime} \circ *^{\prime}\right)\left(\beta_{e e}(m)\right)=\left(\dagger^{\prime} \circ p^{\prime}\right)\left(\beta_{e e}(m)\right)=p^{\prime} \circ \beta_{e e}(m)
$$

For the second equation we compute:

$$
\begin{aligned}
& \beta_{e}(-2 \lambda(a, a),-2 a)=p^{\prime} \circ \beta_{e e}(-2 \lambda(a, a))+\mu^{\prime} \circ \alpha(-2 a)= \\
& -2\left(p^{\prime} \circ \lambda^{\prime}(\alpha(a), \alpha(a))\right)+\mu^{\prime} \circ \alpha(-2 a)= \\
& -2\left(\mu^{\prime}(\alpha(a)+\alpha(a))-\mu^{\prime}(\alpha(a))-\mu^{\prime}(\alpha(a))\right)+\mu^{\prime}(-2 \alpha(a))= \\
& -2\left(4 \mu^{\prime}(\alpha(a))-2 \mu^{\prime}(\alpha(a))\right)+4 \mu^{\prime}(\alpha(a))=-4 \mu^{\prime}(\alpha(a))+4 \mu^{\prime}(\alpha(a))=0
\end{aligned}
$$

We used Lemma 24 for $n= \pm 2$ and hence the commutativity of $\mathfrak{M}$. 
4.6. Symmetric quadratic groups and forms. The simplest case of a quadratic group is where both $*$ and $\dagger$ are trivial. Let's call such a quadratic group $\mathfrak{M}=\left(M_{e} \stackrel{h}{\rightarrow} M_{e e} \stackrel{p}{\rightarrow} M_{e}\right)$ symmetric. Equivalently, this means that $h p=2 \mathrm{id}=p h$ (and hence $M_{e}$ is commutative). Then a quadratic form $(\lambda, \mu): A \rightarrow \mathfrak{M}$ will automatically be symmetric in the sense that

$$
\lambda\left(a, a^{\prime}\right)=\lambda\left(a^{\prime}, a\right) \quad \text { and } \quad \mu(-a)=\mu(a) \quad \forall a \in A .
$$

We call $\mu$ a symmetric quadratic refinement of $\lambda$ and obtain a category of symmetric quadratic forms with a forgetful functor $R$ to the category of symmetric forms. It is not hard to show that the construction in Theorem 25 gives a universal symmetric quadratic refinement $\mu_{\lambda}^{c}$ for any given symmetric bilinear form $\lambda$. More precisely,

Theorem 26. Any symmetric bilinear form $\lambda$ has a universal symmetric quadratic refinement. In fact, the forgetful functor $R(\lambda, \mu)=\lambda$ from the category SQF of symmetric quadratic forms to the category SF of symmetric forms has a left adjoint $L: \mathrm{HF} \rightarrow \mathrm{CQF}, L(\lambda)=\left(\lambda, \mu_{\lambda}^{c}\right)$.

Remark 27. We observe that the map $p_{\lambda}^{c}: M_{e e} \rightarrow M_{e}^{c}$ is a monomorphism in this easiest, symmetric, case, just like it was in the hardest, non-commutative, case. This can be seen by noting that the first set of relations $\left(m^{*}, 0\right)=(m, 0)$ is redundant if the involution $*$ is trivial. Therefore, if $0=p_{\lambda}^{c}(m)=[m, 0]$ then $(m, 0)$ must come from the second set of relations, i.e. it must be of the form

$$
(m, 0)=(-2 \lambda(a, a),-2 a) \quad \text { for some } a \in A .
$$

This implies that $2 a=0$ and hence $\lambda(2 a, a)=0$ which in turn means $m=0$.

Corollary 28. There is an exact sequence

$$
1 \longrightarrow M_{e e} \stackrel{p}{\longrightarrow} M_{e}^{c} \longrightarrow \mathbb{Z}_{2} \otimes A \longrightarrow 1
$$

Examples 29. If $M_{e e}=M_{e}$ then $h=$ id and $p=2$ id is a canonical choice for which $\mu$ is determined by $\lambda$. Another canonical choice is $p=\mathrm{id}$ and $h=2 \mathrm{id}$. Then a quadratic refinement of $\left(M_{e}, h, p\right)$ with this choice exists exactly for even forms, at least for free groups $A$. Moreover, if $M_{e e}$ has no 2-torsion then a quadratic refinement is uniquely determined by the given even form.

At the other extreme, consider $M_{e e}=M_{e}=\mathbb{Z}_{2}$. If $A$ is a finite dimensional $\mathbb{Z}_{2}$-vectorspace then non-singular symmetric bilinear forms $\lambda$ are classified by their rank and their parity, i.e. whether they are even or odd, or equivalently, whether they admit a quadratic refinement or not. In the even case, quadratic forms $(\lambda, \mu)$ are classified by rank and Arf invariant. This additional invariant takes values in $\mathbb{Z}_{2}$ and vanishes if and only if $\mu$ takes more elements to zero than to one (thus the Arf invariant is sometimes referred to as the "democratic invariant").

If $\lambda$ is odd then the following trick allows one to still define Arf invariants and it motivates the introduction of $M_{e}$. Let again $A$ be a finite dimensional $\mathbb{Z}_{2}$-vectorspace, $M_{e e}=\mathbb{Z}_{2}$ and $M_{e}=\mathbb{Z}_{4}$ with the unique nontrivial homomorphisms $h, p$. Then any non-singular symmetric bilinear form $\lambda$ has a quadratic refinement $\mu$ and quadratic forms $(\lambda, \mu)$ are classified by rank and an Arf invariant with values in $\mathbb{Z}_{8}$. If $\lambda$ is even, this agrees with the previous Arf invariant via the linear inclusion $\mathbb{Z}_{2} \subset \mathbb{Z}_{8}$. 
4.7. Presentations for universal quadratic groups. Consider a central group extension

$$
1 \rightarrow M \rightarrow G \stackrel{\pi}{\rightarrow} A \rightarrow 1
$$

and assume that $M$ and $A$ have presentations $\left\langle m_{i} \mid n_{j}\right\rangle$ respectively $\left\langle a_{k} \mid b_{\ell}\right\rangle$. To avoid confusion, we write groups multiplicatively for a while and switch back to additive notation when returning to hermitian forms.

It is well known how to get a presentation for $G$ : Pick a section $s: A \rightarrow G$ with $s(1)=1$ which is not necessarily multiplicative. Write a relation in $A$ as $b_{\ell}=a_{1}^{\prime} \cdots a_{r}^{\prime}$, where $a_{i}^{\prime}$ are generators of $A$ or their inverses, then

$$
1=s(1)=s\left(b_{\ell}\right)=s\left(a_{1}^{\prime}\right) \cdots s\left(a_{r}^{\prime}\right) w_{\ell}
$$

where $w_{\ell}=w_{\ell}\left(m_{i}\right)$ is a word in the generators of $M$. This equation follows from the fact that the projection $\pi$ is a homomorphism and for simplicity we have identified $M$ with its image in $G$. We obtain the presentation

$$
G=\left\langle m_{i}, \alpha_{k} \mid n_{j},\left[m_{i}, \alpha_{k}\right], \beta_{\ell} w_{\ell}\right\rangle
$$

where $\alpha_{k}:=s\left(a_{k}\right)$ and $\beta_{\ell}:=s\left(a_{1}^{\prime}\right) \cdots s\left(a_{r}^{\prime}\right)$ is the same word in the $\alpha_{k}$ as $b_{\ell}$ is in the $a_{k}$. The commutators $\left[m_{j}, \alpha_{k}\right]$ arise because we are assuming that the extension is central, in a more general case one would write out the action of $A$ on $M$.

It will be useful to rewrite this presentation as follows. Observe that the section $s$ satisfies

$$
s\left(a_{1} a_{2}\right)=s\left(a_{1}\right) s\left(a_{2}\right) c\left(a_{1}, a_{2}\right)
$$

for a uniquely determined cocycle $c: A \times A \rightarrow M$. By induction one shows that

$$
\begin{aligned}
& s\left(a_{1} \cdots a_{r}\right)=s\left(a_{1} \cdots a_{r-1}\right) s\left(a_{r}\right) c\left(a_{1} \cdots a_{r-1}, a_{r}\right)=\cdots= \\
& s\left(a_{1}\right) \cdots s\left(a_{r}\right) c\left(a_{1}, a_{2}\right) c\left(a_{1} a_{2}, a_{3}\right) c\left(a_{1} a_{2} a_{3}, a_{4}\right) \cdots c\left(a_{1} \cdots a_{r-1}, a_{r}\right)
\end{aligned}
$$

Comparing this expression with the definition of the word $w_{\ell}$ in the presentation of $G$, it follows that

$$
w_{\ell}=c\left(a_{1}^{\prime}, a_{2}^{\prime}\right) c\left(a_{1}^{\prime} a_{2}^{\prime}, a_{3}^{\prime}\right) \cdots c\left(a_{1}^{\prime} \cdots a_{r-1}^{\prime}, a_{r}^{\prime}\right) \in M
$$

so that the above presentation of $G$ is entirely expressed in terms of the cocycle $c$ (and does not depend on the section $s$ any more).

Now assume that $\lambda: A \times A \rightarrow M$ is a hermitian form with respect to an involution $*$ on $M$. Then the universal (non-commutative) quadratic group $M_{e}$ from Example 18 is a central extension as above with cocycle $c=\lambda$. Reverting to additive notation, we see that

$$
\begin{aligned}
w_{\ell} & =\lambda\left(a_{1}^{\prime}, a_{2}^{\prime}\right)+\lambda\left(a_{1}^{\prime}+a_{2}^{\prime}, a_{3}^{\prime}\right)+\cdots+\lambda\left(a_{1}^{\prime}+\cdots+a_{r-1}^{\prime}, a_{r}^{\prime}\right) \\
& =\sum_{1 \leq i<j \leq r} \lambda\left(a_{i}^{\prime}, a_{j}^{\prime}\right)
\end{aligned}
$$

where the ordering of the summands is irrelevant because $M$ is central in $M_{e}$. Summarizing the above discussion, we get.

Lemma 30. The universal (non-commutative) quadratic group $M_{e}$ corresponding to the hermitian form $\lambda$ has a presentation

$$
M_{e}=\left\langle m_{i}, \alpha_{k} \mid n_{j},\left[m_{i}, \alpha_{k}\right], \beta_{\ell}+\sum_{1 \leq i<j \leq r} \lambda\left(a_{i}^{\prime}, a_{j}^{\prime}\right)\right\rangle
$$


where the generators $m_{i}, \alpha_{k}$ and words $n_{j}, \beta_{\ell}$ are defined as above. Moreover, the universal quadratic refinement $\mu: A \rightarrow M_{e}$ is a (in general non-multiplicative) section of the central extension and hence $\alpha_{k}=\mu\left(a_{k}\right)$ for the generators $a_{k}$ of $A$.

As discussed in Theorem 25, we get the universal commutative quadratic group $M_{e}^{c}$ for $\lambda$ by adding the relations $\left(m^{*}, 0\right)=(m, 0)$ and $(-2 \lambda(a, a),-2 a)=0$. The latter can be rewritten in the form $2(0, a)=(\lambda(a, a), 0)$. In the current notation, where $(m, 0)$ is identified with $m \in M$, we obtain the relations

$$
m^{*}=m \quad \text { and } \quad 2 \mu(a)=\lambda(a, a) \in M_{e}^{c} \quad \forall m \in M, a \in A .
$$

Recalling that $A, M$ and $M_{e}^{c}$ are commutative groups, we can write our presentation in that category to obtain

Lemma 31. The universal (commutative) quadratic group $M_{e}^{c}$ corresponding to the hermitian form $\lambda: A \times A \rightarrow M$ has a presentation

$$
M_{e}^{c}=\left\langle m_{i}, \mu\left(a_{k}\right) \mid n_{j}, \beta_{\ell}+\sum_{1 \leq i<j \leq r} \lambda\left(a_{i}^{\prime}, a_{j}^{\prime}\right), m^{*}=m, 2 \cdot \mu(a)=\lambda(a, a)\right\rangle
$$

Here $\left\langle m_{i} \mid n_{j}\right\rangle$ is a presentation of $M$ and $a_{k}$ are generators of $A$. Moreover, for every relation $b_{\ell}=\sum_{i=1}^{r} a_{i}^{\prime}$ in $A$, we use the word $\beta_{\ell}:=\sum_{i=1}^{r} \mu\left(a_{i}^{\prime}\right)$.

4.8. Twisted intersection invariants and a universal quadratic group. If we apply this construction to the universal inner product on order $n$ rooted trees

$$
\langle,\rangle: \mathcal{L}_{n}(m) \times \mathcal{L}_{n}(m) \longrightarrow \mathcal{T}_{2 n}(m)=: \mathcal{T}_{2 n}(m)_{e e}
$$

we obtain a universal symmetric quadratic refinement

$$
q:=\mu_{\langle,\rangle}^{c}: \mathcal{L}_{n}(m) \rightarrow \mathcal{T}_{2 n}(m)_{e}^{c}
$$

Let us compute the presentation from Lemma 31 in this case. Recall that the generators of $\mathcal{L}_{n}(m)$ are rooted trees $J$ of order $n$ and the relations are the AS and IHX relations from Figure 5. Similarly, $\mathcal{T}_{2 n}(m)$ is generated by unrooted trees $t$ of order $2 n$, modulo the same relations. Putting these together, we see that $\mathcal{T}_{2 n}(m)_{e}^{c}$ is generated by unrooted trees $t$ of order $2 n$ and elements $q(J)$, one for each rooted tree $J$ of order $n$. The three types of relations from Lemma 31 are:

$n_{j}$ : Relations in $M=\mathcal{T}_{2 n}(m)$ are ordinary AS and IHX relations for unrooted trees $t$,

$\beta_{\ell}$ : Every relation $b_{\ell}$ in $A=\mathcal{L}_{n}(m)$ is an AS-relation $J+\bar{J}=0$ or an IHX-relation $I-H+X=0$. We obtain the following twisted AS- respectively IHX-relations:

$$
\begin{aligned}
& 0=q(J)+q(\bar{J})+\langle J, \bar{J}\rangle \\
& 0=q(I)+q(H)+q(X)-\langle I, H\rangle+\langle I, X\rangle-\langle H, X\rangle
\end{aligned}
$$

$c: 2 \cdot q(J)=\langle J, J\rangle$

The last relation $c$ builds in the commutativity of the universal group as discussed above because we are in the easiest, symmetric, setting where the involution $*$ is trivial. Using relation $c$, the twisted AS relation simply becomes

$$
q(\bar{J})=\underset{17}{q(-J)}=q(J)
$$


which was expected since we are in the symmetric case. This relation means that the orientation of $J$ is irrelevant when forming $q(J)$ and in fact, with some care one can see that the twisted IHX-relation makes sense for unoriented trees.

Lemma 32. This is a presentation for the target group $\mathcal{T}_{2 n}^{\infty}(m)$ of twisted Whitney towers from Definition 8 .

Proof. The translation comes from setting $J^{\infty}=q(J)$ for rooted trees $J$ (and keeping unrooted trees unchanged). We need to show that the twisted IHX-relations in the original definition of $\mathcal{T}_{2 n}^{\infty}(m)$ are equivalent to the twisted IHX-relations above, all other relations were already shown to agree. This is very easy to see in the presence of the interior-twist relations: Together with the (untwisted) IHX-relations, they imply that

$$
0=\langle I, I-H+X\rangle=\langle I, I\rangle-\langle I, H\rangle+\langle I, X\rangle=2 \cdot q(I)-\langle I, H\rangle+\langle I, X\rangle
$$

This last expression is exactly the difference between the two versions of the twisted IHXrelations.

Corollary 33. There is an isomorphism of symmetric quadratic groups

$$
\mathcal{T}_{2 n}(m)_{e}^{c} \cong \mathcal{T}_{2 n}^{\infty}(m)
$$

which is the identity on $\mathcal{T}_{2 n}(m)$ and takes $q(J)$ to $J^{\text {co }}$ for rooted trees $J$. The quadratic group structure on $\mathcal{T}_{2 n}^{\infty}(m)$ is given by the homomorphisms $\mathcal{T}_{2 n}(m) \stackrel{p}{\rightarrow} \mathcal{T}_{2 n}^{\infty}(m) \stackrel{h}{\rightarrow} \mathcal{T}_{2 n}(m)$ which are uniquely characterized (for unrooted trees $t$ and rooted trees $J$ ) by

$$
p(t)=t \quad \text { and } \quad h(t)=2 \cdot t, h\left(J^{\infty}\right)=\langle J, J\rangle
$$

Note that Theorem 9 is now a direct consequence of Corollary 28 .

\section{REFERENCES}

[1] H. Baues, Quadratic functors and metastable homotopy, J. Pure Appl. Algebra, vol. 91 (1994) 49-107.

[2] H. Baues, On the group of homotopy equivalences of a manifold. Trans. A. M. S. 348 (1996) 47374773.

[3] J. Conant, R. Schneiderman, P. Teichner, Higher-order intersections in low-dimensional topology, Proceedings of the National Academy 2011, 108 (20) 8131-8138.

[4] J. Conant, R. Schneiderman, P. Teichner, Whitney tower concordance of classical links, preprint (2012) arXiv:1202.3463v1 [math.GT].

[5] S Donaldson, An application of gauge theory to the topology of 4-manifolds, J. Diff. Geom. 18 (1983), $279-315.2$

[6] M. Freedman, R. Kirby, A geometric proof of Rochlin's theorem, Proc. Symp. Pure. Math. 32, A.M.S 1978, 85-98.

[7] M. Freedman, F. Quinn, Topology of 4-manifolds, Princeton Univ. Press.

[8] R. Fintushel, B. Park, R. Stern, Reverse engineering small 4-manifolds, Algebr. Geom. Topol. 7 (2007), 2103-2116.

[9] M. Kervaire, J. Milnor, On 2-spheres in 4-manifolds, Proc. Nat. Acad. USA 47 (1961), 1651-1657.

[10] R. Kirby The topology of 4-manifolds, Springer-Verlag, Berlin, New York, (1989).

[11] A. Ranicki, Algebraic Poincaré cobordism, Topology, geometry, and algebra: interactions and new directions (Stanford, CA, 1999), Contemp. Math., 279, A.M.S., 213-255.

[12] R. Schneiderman, P. Teichner, Higher order intersection numbers of 2-spheres in 4-manifolds, Alg. and Geom. Topology 1 (2001) 1-29.

[13] R. Stong, Existence of $\pi_{1}$-negligible embeddings in 4-manifolds. A correction to Theorem 10.5 of Freedmann and Quinn, Proc. of the A. M.S. 120 (1994) 1309-1314.

[14] C.T.C. Wall, Surgery on compact manifolds, Acad. Press (1970). 
E-mail address: jconant@math.utk.edu

Dept. of Mathematics, University of Tennessee, Knoxville, TN

E-mail address: robert.schneiderman@lehman.cuny.edu

Dept. of Mathematics and Computer Science, lehman College, City University of New YORK, BRONX, NY

E-mail address: teichner@mac.com

Dept. of Mathematics, University of California, Berkeley, CA and

Max-Planck Institut für Mathematik, Bonn, Germany 